\title{
Serum miR-20a is a promising biomarker for gastric cancer
}

\author{
RUIRUI YANG ${ }^{1,2^{*}}$, YUN FU $^{1,2^{*}}$, YING ZENG $^{3 *}$, MENGQIN XIANG ${ }^{1,4}$, YUFANG YIN $^{5}$, \\ $\mathrm{LI} \mathrm{LI}^{1,2}$, HAIFAN XU ${ }^{6}$, JING ZHONG ${ }^{7}$ and XI ZENG ${ }^{1,2,8}$
}

\begin{abstract}
${ }^{1}$ Hunan Province Key Laboratory of Tumor Cellular and Molecular Pathology, Cancer Research Institute, University of South China; ${ }^{2}$ Hunan Province Cooperative Innovation Center for Molecular Target New Drug Study, University of South China; ${ }^{3}$ School of Nursing, University of South China, Hengyang, Hunan 421001; ${ }^{4}$ Department of Pathology, The Hunan Provincial People's Hospital, Changsha, Hunan 410005, P.R. China; ${ }^{5}$ Department of Pharmacology, Southern Illinois University School of Medicine, Springfield, IL 62702, USA; ${ }^{6}$ Department of Surgical Oncology and ${ }^{7}$ Institute of Clinical Medicine, The First Affiliated Hospital, University of South China; ${ }^{8}$ Center for Gastric Cancer Research of Hunan Province, University of South China, Hengyang, Hunan 421001, P.R. China
\end{abstract}

Received November 8, 2016; Accepted January 12, 2017

DOI: $10.3892 /$ br.2017.862

\begin{abstract}
Gastric cancer (GC) is a common type of cancer, particularly in China. Numerous studies have demonstrated that circulating microRNAs (miRNAs) have potential applications as noninvasive biomarkers for cancer diagnosis and prognosis. Microarray-based serum miRNA profiling was performed on the serum of 12 paired pre- and post-operative GC patients to screen differentially expressed serum miRNAs. Twelve different serum miRNAs between pre- and post-operative GC patients were identified. Those miRNAs were verified by real-time quantitative polymerase chain reaction in 110 paired pre- and post-operative serum samples from 55 GC patients. miR-20a was confirmed and demonstrated potential as a GC-associated biomarker. Furthermore, the levels of serum miR-20a were significantly different between GC, nasopharyngeal cancer, colorectal carcinoma, breast cancer and non-cancerous controls. In addition, it was found that serum miR-20a levels correlated with age, tumor stage, differentiated degree and lymph node metastasis in GC. Survival analysis indicated that GC patients with elevated levels of serum miR-20a had poor survival. Thus, serum miR-20a may serve as a molecular marker for diagnosis, evaluating therapeutic efficacy and prognosis, as well as monitoring recurrence in GC patients.
\end{abstract}

Correspondence to: Mr. Xi Zeng, Hunan Province Key Laboratory of Tumor Cellular and Molecular Pathology, Cancer Research Institute, University of South China, 28 West Changsheng Road, Hengyang, Hunan 421001, P.R. China

E-mail: zx397@126.com

*Contributed equally

Key words: gastric cancer, serum, miR-20a, biomarker

\section{Introduction}

Although the incidence of gastric cancer (GC) is declining overall, it remains the fifth most common type of cancer and is the third leading cause of cancer-associated mortality worldwide (1). In 2012, China accounted for $>40 \%$ of novel gastric cancer cases and associated mortalities (1). Patients with GC were often diagnosed at a late stage and had a low survival rate. The 5-year overall survival rate of gastric cancer is closely associated with neoplasm staging. Stage I patients have a 5-year survival rate of $>90 \%$, compared with $<5 \%$ in stage IV patients (2). Therefore, early diagnosis and treatment are crucial to improving the prognosis of GC patients.

Circulating microRNAs (miRNAs) are considered to be ideal as disease biomarkers (3). Recent studies demonstrated that circulating miRNAs have potential as molecular tools for detecting, predicting and monitoring various types of cancer (4-6). Circulating miRNAs may be encapsulated in exosomes or bound to proteins and lipoproteins, increasing their stability in circulating blood and protecting them from endogenous RNAses (7). In addition, the levels of serum or plasma miRNAs are similar in healthy individuals. The levels of circulating miRNAs alter with the development and progression of different types of cancer (8). Therefore, these characteristics give circulating miRNAs great potential as molecular tumor markers.

Tsujiura et al (9) first reported that miRNAs were stable and detectable in all GC plasma samples, and that detection of circulating miRNAs may provide novel complementary tumor markers for GC. Circulating miRNAs may also be used to predict metastasis, early recurrence and poor prognosis in stomach cancer. Imaoka et al (10) found that serum miR-203 had the potential to serve as a noninvasive molecular marker for predicting metastases and prognosis. As the release of circulating miRNA from donor cells and its uptake in recipient cells is not fully understood there are few studies regarding the association between circulating miRNAs and GC treatment. However, certain studies have shown that circulating miRNAs could be used to treat cancer. At present, treatment 
focuses on delivering antisense miRNAs or tumor suppressive miRNAs into target tumor cells via microvesicles (MVs) or exosomes (11). Fonsato et al (12) revealed that delivering anti-miRNAs (miR-451, miR-223, miR-24, miR-125b and miR-31) by MVs derived from human adult liver stem cells inhibits tumor growth induced in severe combined immunodeficiency mice with primary hepatocellular carcinomas (12).

\section{Materials and methods}

Serum samples. The study was approved by the Ethics Committee of the University of South China (Hengyang, China). Informed consent was obtained from all participants included in the study. Serum samples (1-2 ml; n=194) were collected from 127 individuals, including 67 paired pre- and post-operative GC patients, and 12 cases of each of GC, nasopharyngeal cancer (NPC), colorectal carcinoma (CRC), breast cancer (BC) and non-cancerous controls (NC). All the malignant tumor patients were diagnosed by two independent professors of pathology. The tumor serum samples were collected from patients who had not undergone therapy, such as radiotherapy or chemotherapy. The paired post-operative GC blood specimens were obtained on the seventh day post-surgery. The individuals with no evidence of carcinoma were selected as the NC subjects. The serum samples were collected from the consenting individuals from the First Hospital Affiliated of University of South China and Second Hospital Affiliated of University of South China from February 2012 to July 2013, according to our previously published protocol (13). According to AJCC cancer staging manual, each GC patient was staged (14).

Serum treatment and RNA extraction. Serum samples (4 ml venous blood) were collected in the morning before breakfast from all of the study subjects. Samples were incubated at room temperature $\left(15-25^{\circ} \mathrm{C}\right)$ for $1 \mathrm{~h}$ and centrifuged at $1,200 \mathrm{x} \mathrm{g}$ at $4^{\circ} \mathrm{C}$ for $10 \mathrm{~min}$. The upper phase of the serum was collected and stored in a refrigerator at $-80^{\circ} \mathrm{C}$. For the RNA isolation, a human serum sample (volume, $200 \mu \mathrm{l}$ ) was used for extracting total RNA with an miRNeasy Serum/Plasma kit (Qiagen $\mathrm{GmbH}$, Hilden, Germany) according to the manufacturer's protocol. During the RNA extraction, cel-miR-39 was spiked at a fixed concentration and served as an internal control. The total RNA was eluted in $12 \mu \mathrm{l}$ RNase-free water. Finally, the reverse transcription (RT) reaction was prepared according to the instructions of the miScript II RT kit (Qiagen $\mathrm{GmbH}$ ). The template cDNA was removed, according to the manufacturer's protocol, and diluted to $200 \mu 1$ with RNase-free water for use in the quantitative polymerase chain reaction (qPCR).

Serum miRNAs profiling assay. Six paired pre- and post-operative GC serum samples were mixed at the same volume $(50 \mu \mathrm{l})$ for microarray analysis using miScript miRNA PCR Array (MIHS-106ZC; Qiagen GmbH), which contains 84 circulating miRNAs associated with human cancer. The experiment was repeated once with six alternative paired pre- and post-operative GC serum samples.

$R T-q P C R$ and data analysis. Serum samples $(\mathrm{n}=170)$ from GC, NPC, CRC, BC and NC were used for the validation of
Table I. Differentially expressed serum miRNAs screened by microarray in paired pre- and post-operative GC samples.

\begin{tabular}{lccc}
\hline & & \multicolumn{2}{c}{$\begin{array}{c}\text { Fold-change } \\
\text { (pre- vs. }\end{array}$} \\
& $\begin{array}{c}\text { Expression in } \\
\text { post-operative } \\
\text { GC patients }\end{array}$ & post-operative) \\
\cline { 3 - 4 } Gene name & Microarray 1 & Microarray 2 \\
\hline hsa-miR-15b-5p & Downregulated & -2.60 & -3.34 \\
hsa-miR-20a-5p & Downregulated & -3.58 & -3.25 \\
hsa-miR-29a-3p & Downregulated & -45.89 & -3.34 \\
hsa-miR-30a-5p & Downregulated & -7.78 & -3.34 \\
hsa-miR-30c-5p & Downregulated & -2.79 & -2.04 \\
hsa-miR-30e-5p & Downregulated & -11.16 & -9.78 \\
hsa-miR-130a-3p & Downregulated & -2.60 & -3.01 \\
hsa-miR-130b-3p & Downregulated & -3.36 & -2.95 \\
hsa-miR-181b-5p & Downregulated & -3.16 & -3.20 \\
hsa-miR-424-5p & Downregulated & -2.11 & -4.63 \\
hsa-let-7d-5p & Downregulated & -4.56 & -3.63 \\
hsa-let-7g-5p & Downregulated & -5.39 & -79.89 \\
\hline
\end{tabular}

miRNA, microRNA; GC, gastric cancer.

Table II. Differences between the expression levels of the 12 miRNAs from 15 cases of paired pre- and post-operative gastric cancer patients.

\begin{tabular}{lccc}
\hline \multicolumn{5}{c}{$\begin{array}{c}\Delta \text { Cq pre-operative - } \\
\Delta \text { Cq post-operative } \\
\text { (mean } \pm \text { SEM) }\end{array}$} & $95 \%$ CI & P-value \\
Gene name & $-0.491 \pm 1.225$ & $(-1.170,0.187)$ & 0.143 \\
hsa-miR-15b-5p & $-1.422 \pm 0.614$ & $(-1.762,-1.082)$ & 0.000 \\
hsa-miR-20a-5p & $-0.256 \pm 0.598$ & $(-0.587,0.075)$ & 0.120 \\
hsa-miR-29a-3p & $-0.157 \pm 1.368$ & $(-0.915,0.600)$ & 0.663 \\
hsa-miR-30a-5p & $-0.251 \pm 0.798$ & $(-0.694,0.191)$ & 0.243 \\
hsa-miR-30c-5p & $-0.123 \pm 0.903$ & $(-0.668,0.422)$ & 0.632 \\
hsa- miR-30e-5p & $-0.607 \pm 1.178$ & $(-1.259,0.046)$ & 0.066 \\
hsa-miR-130a-3p & $-0.423 \pm 0.976$ & $(-0.964,0.117)$ & 0.115 \\
hsa-miR-130b-3p & $-0.726 \pm 1.530$ & $(-1.574,0.121)$ & 0.087 \\
hsa-miR-181b-5p & $-0.361 \pm 0.967$ & $(-0.175,0.896)$ & 0.171 \\
hsa-miR-424-5p & $-1.198 \pm 0.842$ & $(-1.664,-0.732)$ & 0.000 \\
hsa-let-7d-5p & $-1.339 \pm 0.918$ & $(-1.847,-0.830)$ & 0.000 \\
hsa-let-7g-5p & -1.000 \\
\hline
\end{tabular}

CI, confidence interval.

differentially expressed serum miRNAs. Cel-miR-39 served as internal reference controls and RT-qPCR was performed using a miScript SYBR-Green PCR kit (Qiagen $\mathrm{GmbH}$ ), according to the manufacturer's protocol, to quantify specific serum miRNAs. Relative expression of miRNAs was calculated using $\Delta \mathrm{Cq}\left(\Delta \mathrm{Cq}=\mathrm{Cq}_{\text {miRNA }}-\mathrm{Cq}_{\mathrm{Cel-miR-39}}\right)$ and the fold change was calculated using $2^{-\Delta \Delta \mathrm{Cq}}\left(\Delta \Delta \mathrm{Cq}=\Delta \mathrm{Cq}_{\text {sample } 2}-\Delta \mathrm{Cq}_{\text {sample 1 }}\right)(15)$.

Statistical analysis. SPSS 18.0 (SPSS, Inc., Chicago, IL, USA) was used to perform Kaplan-Meier survival analysis. In 

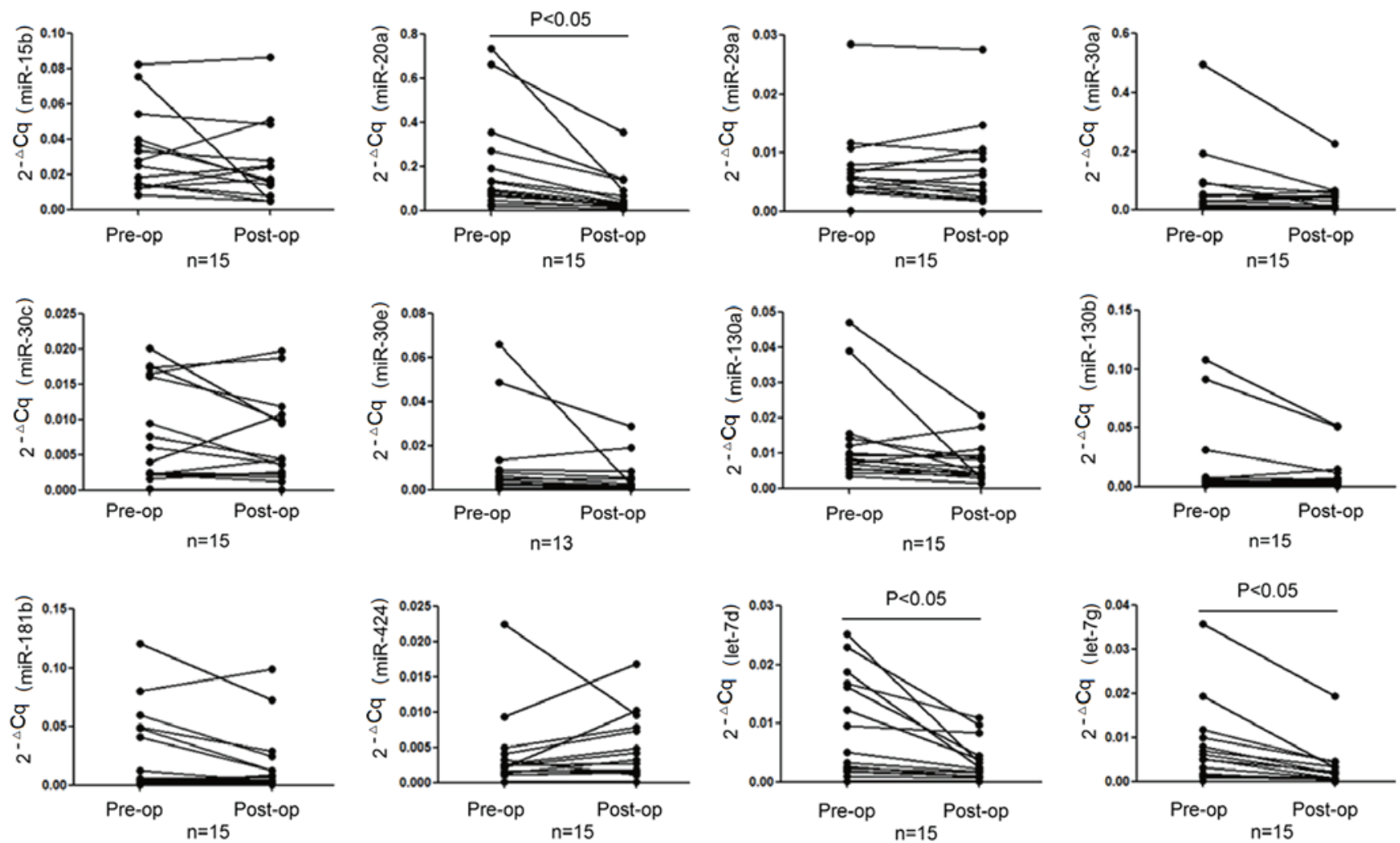

Figure 1. Relative expression levels of 12 miRNAs in 15 cases of paired pre- and post-operative gastric cancer patients. The expression levels of miR-20a, let-7d and let-7g were significantly downregulated in the post-operative group. miRNA, microRNA.

addition Cluster 3.0 (Stanford University, Stanford, CA, USA) and GraphPad Prism 5.0 (GraphPad Software, Inc., La Jolla, CA, USA) software were used. Matched-pairs t-test was used to compare paired pre- and post-operative GC serum samples. The Mann-Whitney U test was used to analyze the difference between two groups, and the differences among three groups were compared using one-way analysis of variance. $\mathrm{P}<0.05$ was considered to indicate a statistically significant difference and all P-values were two-tailed.

\section{Results}

Differentially expressed serum miRNA profile between pre-and post-operative GC patients. The miRNA expression profiling of the serum between pre- and post-operation GC patients was analyzed following preliminary screening with microRNA Array analysis. The principles for screening differentially expressed serum miRNAs are as follows: i) The fold-change of miRNAs in serum between the pre- and post-operative GC samples is $>2$; ii) the miRNA Cq value should be $<35$ in pre- or post-operative GC samples. Based on these principles, the initial screening of the following 12 differentially expressed serum miRNAs between pre- and post-operative GC: miR-15b-5p, miR-20a-5p, miR-29a-3p, miR-30a-5p, miR-30c-5p, miR-30e-5p, miR-130a-3p, miR-130b-3p, miR-181b-5p, miR-424-5p, let-7d-5p, let-7g-5p were subjected to the next step of verification (Table I).

Validation of the miRNAs differentially expressed in paired pre- and post-operative GC serum samples using RT-qPCR. RT-qPCR was performed to verify the 12 miRNAs in the
15 paired pre- and post-operative GC serum samples. The data was analyzed using the relative quantitative method. The expression levels of miR-20a-5p, let-7d-5p and let-7g-5p were identified to be significantly downregulated in the post-operative group $(\mathrm{P}<0.05$; Fig. 1 and Table II). miR-20a-5p, let-7d-5p and let-7g-5p were verified further in the remaining 40 paired GC serum specimens. The results demonstrated that these three serum miRNAs were significantly different between the pre- and post-operative groups $(\mathrm{P}<0.05$, Fig. $2 \mathrm{~A})$; miR-20a-5p, let-7d-5p and let-7g-5p were downregulated in all 55 paired serum samples following surgery. Among these three miRNAs, the difference between the GC patient pre- and post-operative serum miR-20a-5p expression levels was the largest (Fig. 2B and Table III).

Detecting the serum levels of miR-20a-5p in healthy volunteers and in subjects with other common tumors. The expression levels of miR-20a-5p were measured in the serum of 12 cases of each of GC, 12 NPC, 12 CRC, 12 BC and 12 $\mathrm{NC}$. The results indicate that the serum levels of miR-20a-5p in GC, NPC, CRC and BC are significantly higher than the NC $(\mathrm{P}<0.05$; Fig. 3). While no significant difference was identified between the four tumor groups. The present study proposes that serum miR-20a-5p may serve as a novel biomarker to diagnose gastric carcinoma and may also be used to detect NPC, CRC and BC.

Clinical significance of serum miR-20a-5p expression levels in the pre-operative GC patient group. The serum miR-20a-5p expression levels of the 55 GC patients were analyzed prior to surgery. The pre-operative serum was divided into high 
Table III. Differences of miR-20a, let-7d and let-7g between all 55 paired pre- and post-operative gastric cancer serum samples.

\begin{tabular}{lcc}
\hline & $\begin{array}{c}\Delta \text { Cq pre-operative - } \\
\Delta \text { Cq post-operative } \\
\text { Gene name }\end{array}$ & \multicolumn{1}{c}{ (mean \pm SEM) } \\
\hline hsa-miR-20a-5p & $-1.994 \pm 0.700$ & $(-2.183,-1.805)$ \\
hsa-let-7d-5p & $-1.581 \pm 0.645$ & $(-1.756,-1.407)$ \\
hsa-let-7g-5p & $-1.221 \pm 0.885$ & $(-1.459,-0.982)$ \\
\hline
\end{tabular}

CI, confidence interval.

Table IV. Association between the expression levels of serum miR-20a and clinicopathological characteristics of patients.

\begin{tabular}{|c|c|c|c|c|}
\hline \multirow{2}{*}{$\begin{array}{l}\text { Clinical } \\
\text { parameter }\end{array}$} & \multirow[b]{2}{*}{ Nos. } & \multicolumn{2}{|c|}{$\begin{array}{l}\text { Expression level of } \\
\text { serum miR-20a }\end{array}$} & \multirow{2}{*}{$\begin{array}{l}\text { P-value } \\
\text { (2-sided) }\end{array}$} \\
\hline & & Low & High & \\
\hline Gender & & & & 0.396 \\
\hline Male & 35 & 17 & 18 & \\
\hline Female & 20 & 11 & 9 & \\
\hline Age (years) & & & & 0.048 \\
\hline$\geq 60$ & 33 & 15 & 18 & \\
\hline$<60$ & 22 & 13 & 9 & \\
\hline TNM stage (14) & & & & 0.000 \\
\hline I & 19 & 13 & 6 & \\
\hline II & 14 & 6 & 8 & \\
\hline III & 17 & 5 & 12 & \\
\hline IV & 5 & 4 & 1 & \\
\hline Differentiation & & & & 0.000 \\
\hline PDAC & 29 & 16 & 13 & \\
\hline MDAC & 19 & 7 & 12 & \\
\hline WDAC & 7 & 5 & 2 & \\
\hline $\begin{array}{l}\text { Lymph node } \\
\text { metastasis }\end{array}$ & & & & 0.002 \\
\hline Yes & 35 & 15 & 20 & \\
\hline No & 20 & 13 & 7 & \\
\hline Tumor size $(\mathrm{cm})$ & & & & 0.777 \\
\hline$\geq 3$ & 30 & 15 & 15 & \\
\hline$<3$ & 25 & 13 & 12 & \\
\hline
\end{tabular}

PDAC, poorly differentiated adenocarcinoma; MDAC, moderately differentiated adenocarcinoma; WDAC, well differentiated adenocarcinoma.

miR-20a-5p and low miR-20a-5p expression level groups according to the median value, and is presented in combination with the clinicopathological parameters of GC patients in Table IV. Next the concentrations of serum miR-20a-5p was compared among different clinicopathological characteristic groups and the clinical value was evaluated. As shown in Table IV and Fig. 4A, the expression levels of serum miR-20a-5p were significantly associated with age,
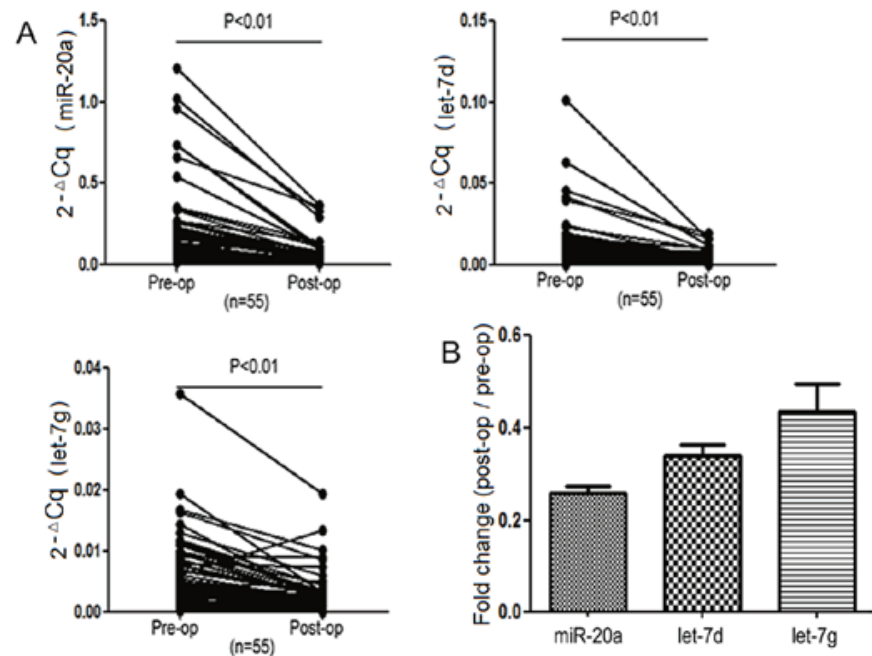

Figure 2. Relative expression levels of miRNAs in paired pre- and post-operative serum samples of gastric cancer patients. The relative expression levels of (A) miR-20a, let-7d and let-7g in 55 paired pre- and post-operative serum samples of gastric cancer patients. (B) Comparison of miR-20a, let-7d and let- $7 \mathrm{~g}$ expression levels in the detected paired pre- and post-operative gastric cancer serum samples. The fold changes (post-operative/pre-operative) are presented.

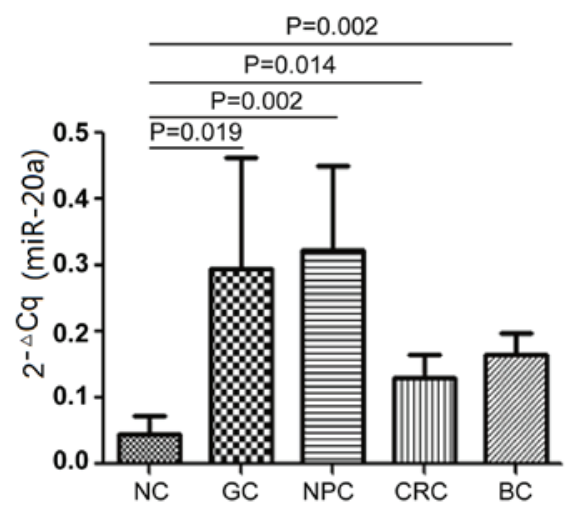

Figure 3. Serum miR-20a expression levels in NC, GC, NPC, CRC and BC (presented as means \pm standard deviation). $\mathrm{NC}$, non-cancer; GC, gastric cancer; NPC, nasopharyngeal cancer; CRC, colorectal cancer; BC, breast cancer.

tumor stage (14), degree of differentiation and lymph node metastasis (Table IV). The serum levels of miR-20a-5p in TNM stage II, III and IV were significantly higher than TNM stage I, with no difference between TNM stages II, III and IV. Concentrations of miR-20a-5p in the well-differentiated group were lower than that of the moderately- and poorly-differentiated groups, whereas no significant difference was noted between the moderately- and poorly-differentiated groups. GC patients with lymph node metastasis exhibited higher miR-20a-5p expression levels than those patients who had no metastasis.

Predictive ability of serum miR-20a-5p levels for GC prognosis. To evaluate the potential prognostic value of miR-20a, The 55 GC patients who had undergone surgery were followed up. At 34 months, 27 cases had succumbed during the process of investigation and the overall survival rate was $50.9 \%$. The results of Kaplan-Meier survival analysis indicate that 

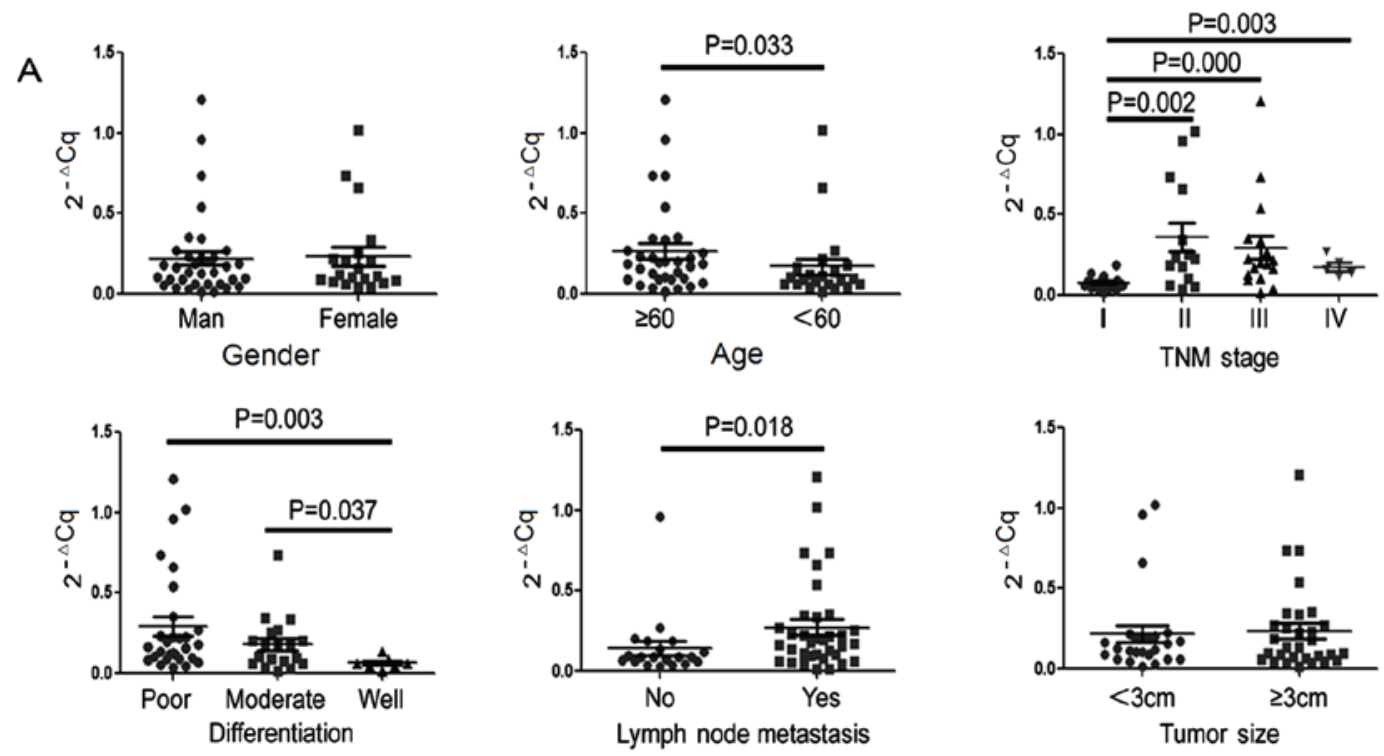

B

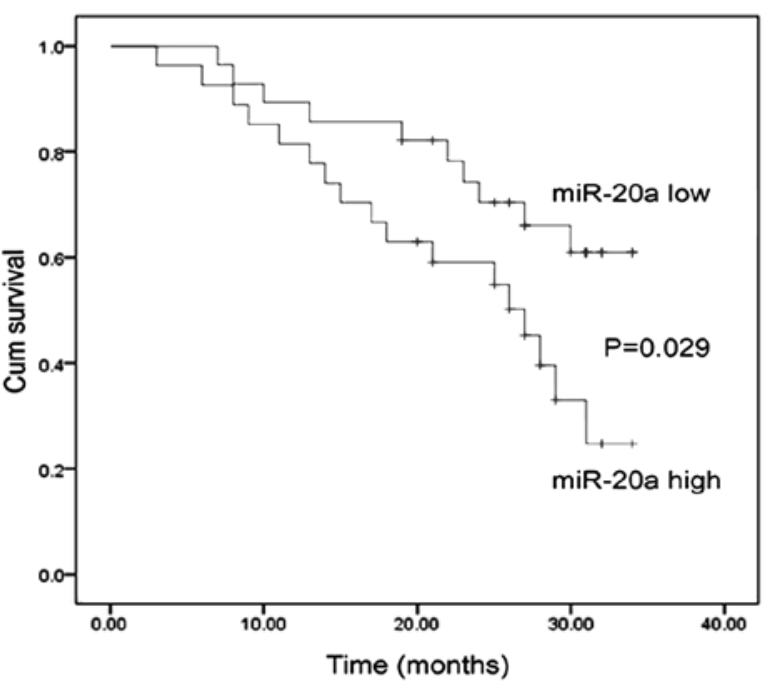

Figure 4. (A) miR-20a expression levels in the serum of the different gastric cancer groups; (B) Kaplan-Meier survival curve analysis of serum miR-20a expression levels.

the overall survival rate of those patients who exhibited low serum miR-20a-5p expression levels was significantly higher than the GC patients who had high serum expression levels of miR-20a-5p (P<0.05; Fig. 4B). Thus, the serum miR-20a-5p expression levels may be used for predicting the prognosis of GC patients.

\section{Discussion}

miR-20a is a member of the miR-17-92 cluster (including miR-17, miR-18a, miR-19a, miR-20a, miR-19b-1 and miR-92a-1), which is commonly upregulated in malignant cancer and involved in carcinogenesis as the classical oncogene (16). Previous studies have reported upregulated expression levels of circulating miR-20a in malignant neoplasms, such as non-small cell lung cancer (NSCLC), and in cervical, hepatoma, colorectal and prostate cancers (17-22). However, the association between the expression level of miR-20a and GC is rarely reported. miR-20a is usually identified by screening for circulating miRNA expression profiles in malignant tumor patients.
Liu et al (23) reported that five serum miRNAs (miR-1, miR-20a, miR-27a, miR-34 and miR-423-5p) had potential in GC diagnosis. In addition, the authors demonstrated that the areas under the receiver operating characteristic curve of these five serum miRNAs (0.879) were markedly higher than those of the classical biomarkers, such as carcinoembryonic antigen (0.503) and cancer antigen 19-9 (0.600) (23). Du et al (24) reported that expression levels of miR-20a were significantly upregulated in GC tissue and plasma samples. Their results indicated that miR-20a may promote activation of the nuclear factor (NF)- $\mathrm{kB}$ signaling pathway and downstream targets, livin and survivin by targeting NF- $\mathrm{\kappa B}$ inhibitor $\beta$ (24).

In the present study, serum miR-20a expression levels in 67 cases of paired pre- and post-operative GC samples were evaluated by microarray and RT-qPCR technology. The use of paired pre- and post-operative GC samples eliminated the individual differences, such as age, gender and eating habits. C. elegans spike-in non-human miR-39 served as an exogenous reference gene in each experiment and RT-qPCR amplification was repeated three times in each 
serum specimen of GC to ensure that the results were highly accurate, repeatable and stable. miR-20a was easily detected and identified to be downregulated following surgery, indicating that the elevated expression levels of serum miR-20a in pre-operative GC patients were released by GC cells. Serum expression levels of miR-20a declined following surgery; therefore, the expression levels may be useful for evaluating the efficacy of GC therapy. In addition, serum miR-20a expression levels were identified to be upregulated in GC, CRC, BC and NPC patients, demonstrating that serum expression levels of miR-20a may be used to diagnose GC and other types of cancer, and that the miR-20a observed in the serum of tumor patients may be released by various types of cancer cell.

In the present study, expression levels of serum miR-20a were found to be significantly associated with age, TNM stage, degree of differentiation and metastasis. Notably, pre-operative GC patients with moderately- or poorly-differentiated cancer cells, or with metastasis exhibited the highest expression levels of serum miR-20a. GC patients that were $\geq 60$ years exhibited higher expression levels of serum miR-20a, which may be due to the age distribution of GC, but this requires further investigation. Patients in the pre-operative group with higher expression levels of serum miR-20a demonstrated a poorer survival rate when compared with GC patients with low expression levels of serum miR-20a. Thus, concentrations of serum miR-20a may be used to predict the prognosis and recurrence of GC.

In conclusion, the present results demonstrated that serum miR-20a expression levels were upregulated in pre-operative GC patients and significantly downregulated in post-operative GC patients. Serum miR-20a could, therefore, serve as a biomarker for diagnosing $\mathrm{GC}$, as well as evaluating the curative effects, predicting the prognosis and monitoring the recurrence of GC patients. The exact efficiency of miR-20a as the tumor marker remains to be confirmed by further validation.

\section{Acknowledgements}

The present study was supported by the National Natural Science Foundation of China (grant no. 81101643); the Hunan Provincial Education Department document (document no. 2014-405); the Foundation of the Construct Program of the Key Discipline in Hunan Province of China (grant no. 2011-76); and the Hunan Province Key Laboratory (grant no. 2016TP1015). The authors would like to thank Dr Chun Wang of Washington University (St. Louis, MO, USA) for assistance with language editing.

\section{References}

1. Torre LA, Bray F, Siegel RL, Ferlay J, Lortet-Tieulent J and Jemal A: Global cancer statistics, 2012. CA Cancer J Clin 65 87-108, 2015

2. Hou X, Zhang M and Qiao H: Diagnostic significance of miR-106a in gastric cancer. Int J Clin Exp Pathol 8: 13096-13101, 2015.

3. Zeng X, Xiang J, Wu M, Xiong W, Tang H, Deng M, Li X, Liao Q, Su B, Luo Z, et al: Circulating miR-17, miR-20a, miR-29c, and miR-223 combined as non-invasive biomarkers in nasopharyngeal carcinoma. PLoS One 7: e46367, 2012.

4. Schwarzenbach H, Nishida N, Calin GA and Pantel K: Clinical relevance of circulating cell-free microRNAs in cancer. Nat Rev Clin Oncol 11: 145-156, 2014.
5. Bianchi F: Lung cancer early detection: the role of circulating microRNAs. EBioMedicine 2: 1278-1279, 2015.

6. Mirzaei HR, Sahebkar A, Mohammadi M, Yari R, Salehi H, Jafari MH, Namdar A, Khabazian E, Jaafari MR and Mirzaei H: Circulating microRNAs in hepatocellular carcinoma: potential diagnostic and prognostic biomarkers. Curr Pharm Des 22: 5257-5269, 2016.

7. Vickers KC and Remaley AT: Lipid-based carriers of microRNAs and intercellular communication. Curr Opin Lipidol 23: 91-97, 2012.

8. Ganepola GA, Rutledge JR, Suman P, Yiengpruksawan A and Chang DH: Novel blood-based microRNA biomarker panel for early diagnosis of pancreatic cancer. World J Gastrointest Oncol 6: 22-33, 2014.

9. Tsujiura M, Ichikawa D, Komatsu S, Shiozaki A, Takeshita H, Kosuga T, Konishi H, Morimura R, Deguchi K, Fujiwara H, et al: Circulating microRNAs in plasma of patients with gastric cancers. Br J Cancer 102: 1174-1179, 2010.

10. Imaoka H, Toiyama Y, Okigami M, Yasuda H, Saigusa S, Ohi M, Tanaka K, Inoue Y, Mohri Y and Kusunoki M: Circulating microRNA-203 predicts metastases, early recurrence, and poor prognosis in human gastric cancer. Gastric Cancer 19: 744-753, 2016.

11. Cheng G: Circulating miRNAs: Roles in cancer diagnosis, prognosis and therapy. Adv Drug Deliv Rev 81: 75-93, 2015.

12. Fonsato V, Collino F, Herrera MB, Cavallari C, Deregibus MC, Cisterna B, Bruno S, Romagnoli R, Salizzoni M, Tetta C, et al: Human liver stem cell-derived microvesicles inhibit hepatoma growth in SCID mice by delivering antitumor microRNAs. Stem Cells 30: 1985-1998, 2012.

13. Xiang M, Zeng Y, Yang R, Xu H, Chen Z, Zhong J, Xie H, Xu Y and Zeng X: U6 is not a suitable endogenous control for the quantification of circulating microRNAs. Biochem Biophys Res Commun 454: 210-214, 2014

14. Edge SB, Byrd DR, Compton CC, Fritz AG, Greene FL and Trotti A (eds): AJCC Cancer Staging Manual. 7th edition. Springer-Verlag, New York, NY, 2009.

15. Livak KJ and Schmittgen TD: Analysis of relative gene expression data using real-time quantitative PCR and the 2(- $\Delta \Delta C(T))$ Method. Methods 25: 402-408, 2001.

16. Jiang Z, Yin J, Fu W, Mo Y, Pan Y, Dai L, Huang H, Li S and Zhao J: miRNA 17 family regulates cisplatin-resistant and metastasis by targeting TGFbetaR2 in NSCLC. PLoS One 9: e94639, 2014

17. Babu KR and Muckenthaler MU: miR-20a regulates expression of the iron exporter ferroportin in lung cancer. J Mol Med (Berl) 94: 347-359, 2016.

18. Safari A, Seifoleslami M, Yahaghi E, Sedaghati $F$ and Khameneie MK: RETRACTED ARTICLE: Upregulation of miR-20a and miR-10a expression levels act as potential biomarkers of aggressive progression and poor prognosis in cervical cancer. Tumour Biol: Oct 1, 2015 (Epub ahead of print).

19. Zhang Y, Zheng L, Ding Y, Li Q, Wang R, Liu T, Sun Q, Yang H, Peng S, Wang W, et al: miR-20a induces cell radioresistance by activating the PTEN/PI3K/Akt signaling pathway in hepatocellular carcinoma. Int J Radiat Oncol Biol Phys 92: 1132-1140, 2015.

20. Sokolova V, Fiorino A, Zoni E, Crippa E, Reid JF, Gariboldi M and Pierotti MA: The effects of miR-20a on p21: two mechanisms blocking growth arrest in TGF- $\beta$-responsive colon carcinoma. J Cell Physiol 230: 3105-3114, 2015.

21. Azizian A, Kramer F, Jo P, Wolff HA, Beißbarth T, Skarupke R, Bernhardt M, Grade M, Ghadimi BM and Gaedcke J: Preoperative prediction of lymph node status by circulating mir-18b and mir-20a during chemoradiotherapy in patients with rectal cancer. World J Surg 39: 2329-2335, 2015.

22. Qiang XF, Zhang ZW, Liu Q, Sun N, Pan LL, Shen J, Li T, Yun C, $\mathrm{Li} \mathrm{H}$ and Shi LH: miR-20a promotes prostate cancer invasion and migration through targeting ABL2. J Cell Biochem 115: 1269-1276, 2014

23. Liu R, Zhang C, Hu Z, Li G, Wang C, Yang C, Huang D, Chen X, Zhang $\mathrm{H}$, Zhuang R, et al: A five-microRNA signature identified from genome-wide serum microRNA expression profiling serves as a fingerprint for gastric cancer diagnosis. Eur J Cancer 47: 784-791, 2011.

24. Du Y, Zhu M, Zhou X, Huang Z, Zhu J, Xu J, Cheng G, Shu Y, Liu P, Zhu W, et al: miR-20a enhances cisplatin resistance of human gastric cancer cell line by targeting NFKBIB. Tumour Biol 37: 1261-1269, 2016. 